\title{
Automatic Detection and Classification of Alzheimer's Disease from MRI using TANNN
}

\author{
Eman M. Ali \\ Department of \\ Computer Science \\ Helwan University \\ Cairo, Egypt
}

\author{
Ahmed F. Seddik \\ Dean of Faculty of \\ Computer Science, \\ Nahda University. \\ Professor at the \\ Biomedical Engineering \\ Department, \\ Helwan University. \\ Cairo, Egypt
}

\author{
Mohamed H. Haggag \\ Professor at Computer \\ Science Department \\ Faculty of Computers \\ and Information, \\ Helwan University \\ Cairo, Egypt
}

\begin{abstract}
Early detection of Alzheimer's disease (AD) is important so that preventative measures can be taken. Current techniques for detecting $\mathrm{AD}$ rely on cognitive impairment testing which unfortunately does not yield accurate diagnoses until the patient has progressed beyond a moderate AD. Alzheimer's disease considered being one of the acute diseases that cause the human death especially in people above 60 years old.
\end{abstract}

Many computer-aided diagnosis systems are now widely spread to aid in Alzheimer diagnosis. Therefore, an automated and reliable computer-aided diagnostic system for diagnosing and classifying the brain diseases has been proposed [1]

MRI (Magnetic resonance Imaging) is one source of brain diseases detection tools, but using MRI in Alzheimer classification is considered to be difficult process according to the variance and complexity of brain tissue. This paper presents a survey of the most famous techniques used for the classification of brain diseases based on MRI [2].

The Alzheimer detection and classification systems consist of four stages, namely, MRI preprocessing, Segmentation, Feature extraction, and Classification stages respectively. In the first stage, the main task is to eliminate the medical resonance images (MRI) noise which may cause due to light reflections or any inaccuracies in the imaging medium.

The second stage, which is the stage where the region of interest is extracted (Alzheimer region). In the third stage, the features related to MRI images will be obtained and stored in an image vector to be ready for the classification process. And finally the fourth stages, where classifier will take place to specify the Alzheimer kind.

TANNN is a new classification technique user to get a very high performance compared with other classification techniques such as KNN, SVM, DT, and Naïve Bayes.

\section{Keywords}

Alzheimer, MRI, Image Classification, Naïve Bayes, Decision Tree, Support Vector Machine, k-Nearest Neighbor.

\section{INTRODUCTION}

In Egypt, over 400 thousand people are afflicted with Alzheimer's disease (approximately 6\% of the population). One in six Egyptian above age 60 is diagnosed with $\mathrm{AD}$, and it is the fourth leading cause of death in Egypt. An absolute diagnosis is not possible until moderate to severe cortex damage has occurred.
Early detection and classification of Alzheimer are very important in, clinical practice. Several researchers have proposed different techniques for the classification of Alzheimer using different sources of information [1]. In this paper, we test the TANNN technique upon three of the real Alzheimer datasets. By analyzing the MR image and Magnetic Resonance Spectroscopy (MRS) a final decision can take place either the data collected from patient with very mild, mild and moderate Alzheimer.

By using image processing techniques a Alzheimer region can be obtained by using a combination of denoising, segmentation, feature extraction and classification techniques. The proposed technique has the potential of assisting medical diagnosis.

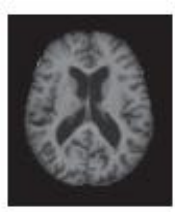

(a)

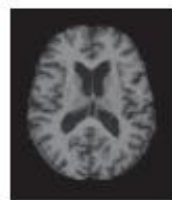

(b)

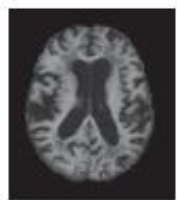

(c)

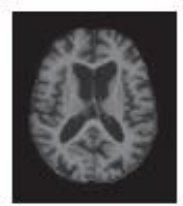

(d)
Fig.1. (a) brain with no dementia (b) brain with a very mild AD (c) brain with a mild AD (d) brain with a moderate AD.

Prevalence of AD doubles every 5 years of life after age 60 . $\mathrm{AD}$ is the most common dementing illness and a major public health issue of increasing importance as life expectancy increases. Although non-invasive approaches for antemortem diagnosis of $\mathrm{AD}$ are under development, definitive diagnosis of $\mathrm{AD}$ requires neuropathologic confirmation of the characteristic amyloid plaques and neurofibrillary tangles [4]. New drugs under development will target different stages of disease pathophysiology, and efficacious AD treatments likely will require early initiation before irreversible brain tissue damage. Thus, a great deal of attention has been paid recently to the prodromal stage of $\mathrm{AD}$, referred to as mild cognitive impairment (MCI), which includes individuals with memory problems who do not meet criteria for dementia. Although MCI definitions vary across studies [4], MCI individuals convert to $\mathrm{AD}$ with rates of $6-15 \%$ annually [4]. Therefore, MCI individuals are a high-risk group likely to benefit from effective treatments see Fig. 1[2]. 


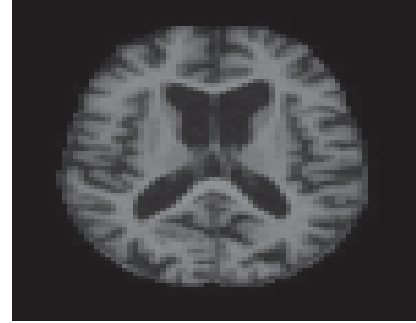

Fig.2. Brain MRI AD region [2].

These two types of Alzheimer are life-threatening and cause death. The growth of the Alzheimer affects the brain because space in the skull is limited. This growth causes the intracranial pressure to be increased and damage the other healthy tissues that perform important functions inside the brain [4-6].

$\mathrm{AD}$ follows a distinct pattern of brain damage as the disease progresses. One of the first affected areas is the hippocampus, which is responsible for episodic and spatial memory in addition to serving as a relay structure between the brain and body. The hippocampus shrinks abnormally in an AD patient; normal reduction per year is between 0.24 and $1.73 \%$, whereas a hippocampus inflicted with AD may shrink anywhere between 2.2 and $5.9 \%$ [1]. The reduction in hippocampal volume is mainly attributed to cell loss and damage, particularly to synapses, or neuron ends. Synapse destruction results in a loss of ability for neurons to communicate via signaling to each other.

Computer technologies are widely used nowadays in medical diagnoses especially cancer-related researches such as the brain, breast, and liver. The usage of computer image processing technologies in medical diagnosis resulted in the use of some techniques for human body organs photos. MRI; is one of the most widely used technologies through the usage of the magnetic field, it has much higher features than other radiation tools such as $\mathrm{x}$-ray and computed tomography (CT) [4].

Various classification techniques are used in classifying Alzheimer in MRI. The statistical, Intensity, Symmetry, Texture features etc., which utilize a gray value of Alzheimer are used here for classifying the Alzheimer. However, the gray values of MRI tend to change due to over-enhancement or in the presence of noise [7-10].

The classification techniques used mainly on prospecting the MR image and extract some statistical or texture from the medical image to learn from how interestingly discern medical images to eventually develop "knowledgeable " computer systems[11][12]. Thus, the objective of this paper presents an appraisal of the existing and conventional methods for the classification of medical images and based on these observations; propose a new framework for medical image classification. The rest of the paper is structured as Section 2 to Section 4.

Section 2 present a brief description of the TANNN, section 3 show the result of applying TANNN on one of the real brain diseases datasets in the world and finally section 4 state the final conclusion.

\section{APPLYING DATA MINING TECHNIQUES ON REAL ALZHEIMER DATASET}

The proposed system has mainly four modules namely Preprocessing, segmentation using Contribution-Based Clustering Algorithm, Feature extraction, and disease classification. According to the need of the next level, the preprocessing step converts the image. It performs filtering of noise and other artifacts in the image and sharpening the edges in the image. RGB to gray conversion and reshaping also takes place here. It includes a median filter for noise removal. The feature extraction is extracting the cluster, which shows the predicted Alzheimer at the Haar wavelet transform output. The extracted cluster is given to the threshold process. It applies a binary mask over the entire image [2].

In the disease detection and classification step, the Alzheimer area is calculated using the binarization method making the dark pixel darker and white brighter. In threshold coding, each transform coefficient is compared with a threshold and if it's less than the threshold value, it is considered as zero or else one. In the approximate reasoning step, the Alzheimer area is calculated using the binarization method. That is the image having only two values either black or white ( 0 or 1$)$. Here 200x200 JPEG image is a maximum image size. The binary image can be represented as a summation of a total number of white and black pixels. Pre-processing is done by filtering [2].

Segmentation is carried out by Content-based Image Retrieval (CBIR) algorithm. The feature extraction is done by considering the threshold and finally, approximating the classification method to recognize the Alzheimer shape and position in MRI image using edge detection method [12] see Fig. 4.

\subsection{TANNN}

Most of Data mining techniques used mainly to kinds of classification methods which are:

\section{Statistical methods \\ 2. Data comparisons methods}

Statistical methods such as Naïve Bayes, SVM and Discriminative Analysis; these methods mainly are very complex in applying and in computation complexity.

Data comparisons method such as Decision Tree, Nearest Neighbour, and Neural Network; these methods are considered to be simple but at the same time it's consumed a lot of time which increase the time complexity in these algorithms.

In comparison with these two methods it's clear that both of these methods are complex so to get a full classification technique a merge in both methods will be more benefit ; it will be faster (compared with the data comparisons methods) and also it will be lower in computation complexity (in comparison with the statistical methods). The proposed technique used to detect and classify $\mathrm{AD}$ types which are:
1. No dementia
2. Very mild AD.
3. Mild AD
4. Moderate AD. 


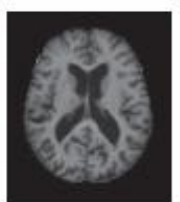

(a)

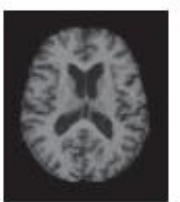

(b)

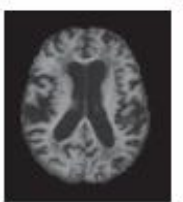

(c)

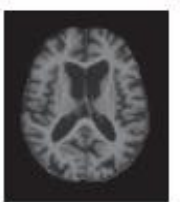

(d)
Fig.3. (a) brain with no dementia (b) brain with a very mild AD (c) brain with a mild AD (d) brain with a moderate AD

\section{EXPREMINTAL RESULTS}

In this section, the results obtained using a database of images is presented. Start by presenting the database with which conducted in tests, and then, present the results according to the used structure.

\subsection{Database}

The famous medical imaging is MRI. A magnetic resonance imaging (MRI) scanner uses powerful magnets to polarize and excite hydrogen nuclei (single proton) in human tissue, which produces a signal that can be detected and it is encoded spatially, resulting in images of the body.

Here we apply the proposed algorithm on one of the real MRI Alzheimerdatasets called BRATS []. Also, we can check the proposed system upon more than three datasets.

\subsubsection{OASIS Dataset [13]}

OASIS is made available by Dr. Randy Buckner at the Howard Hughes Medical Institute (HHMI) at Harvard University, the Neuroinformatics Research Group (NRG) at Washington University School of Medicine, and the Biomedical Informatics Research Network (BIRN). OASIS dataset contains 416 images from infants to 18 years old ages.
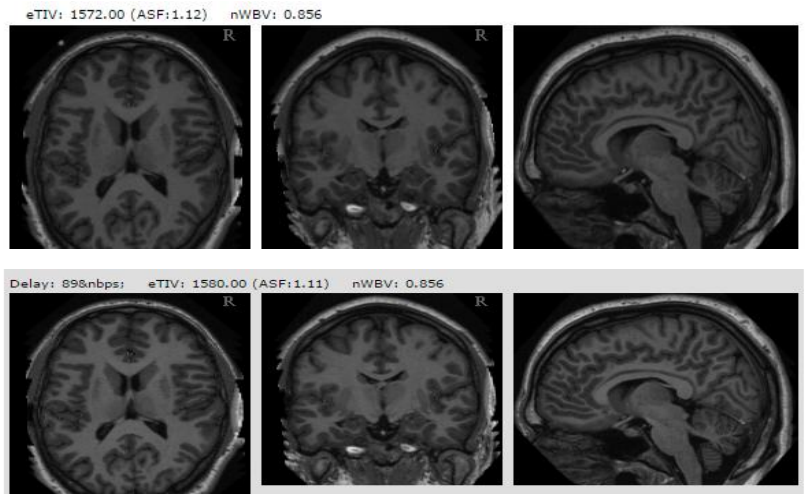

Fig.7. Samples of children brain dataset containing Alzheimer OASIS dataset images [13].

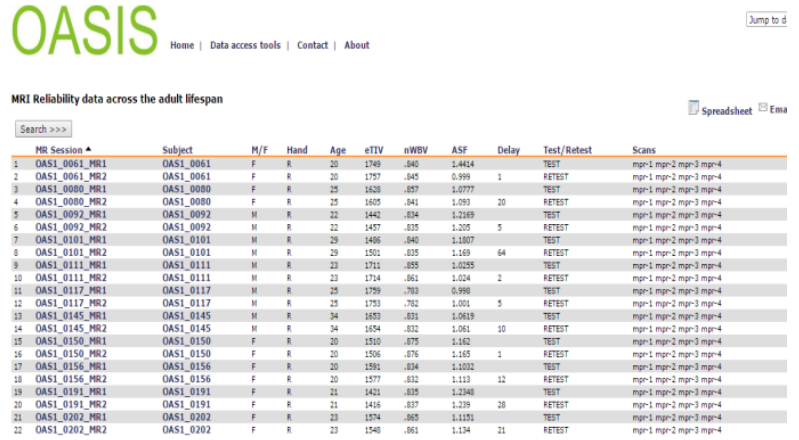

Fig.8. Samples of OASIS dataset data [13].

\subsection{Comparative Analysis}

Next figures show the images as an output. I.e. gray scale image, median filter image, segmented image, finally extracted Alzheimer from MRI image. For this purpose, realtime patient data is taken for analysis. As Alzheimer in MRI image have an intensity more than that of its background so it becomes very easy locate it and extract it from MRI image.

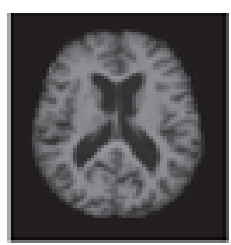

(A)

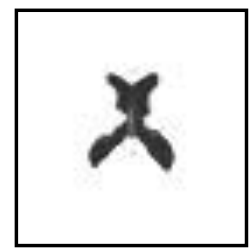

(B)
Fig.9. (A): MRI image of Alzheimer affected brain gray scale image.(B): Threshold segmented image

After using two kinds of segmentation techniques on these images, contribution based information retrieval achieving better performance compared with K-Means algorithm see Fig. 7 and Fig. 8.
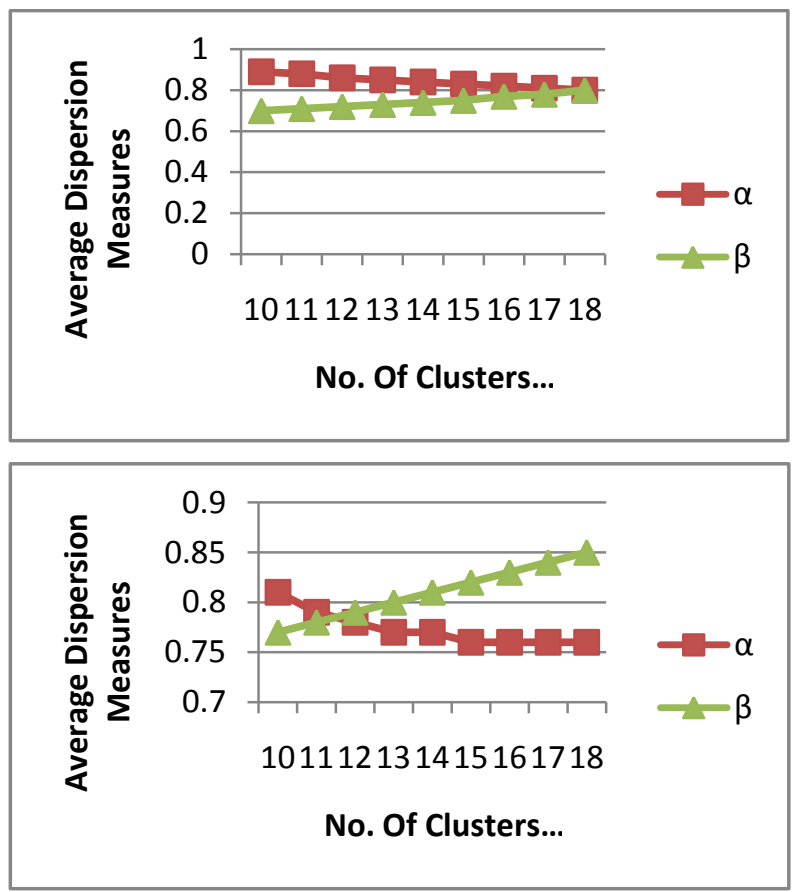

Fig.7. Average value of $\alpha$ and $\beta$ against the number of clusters 

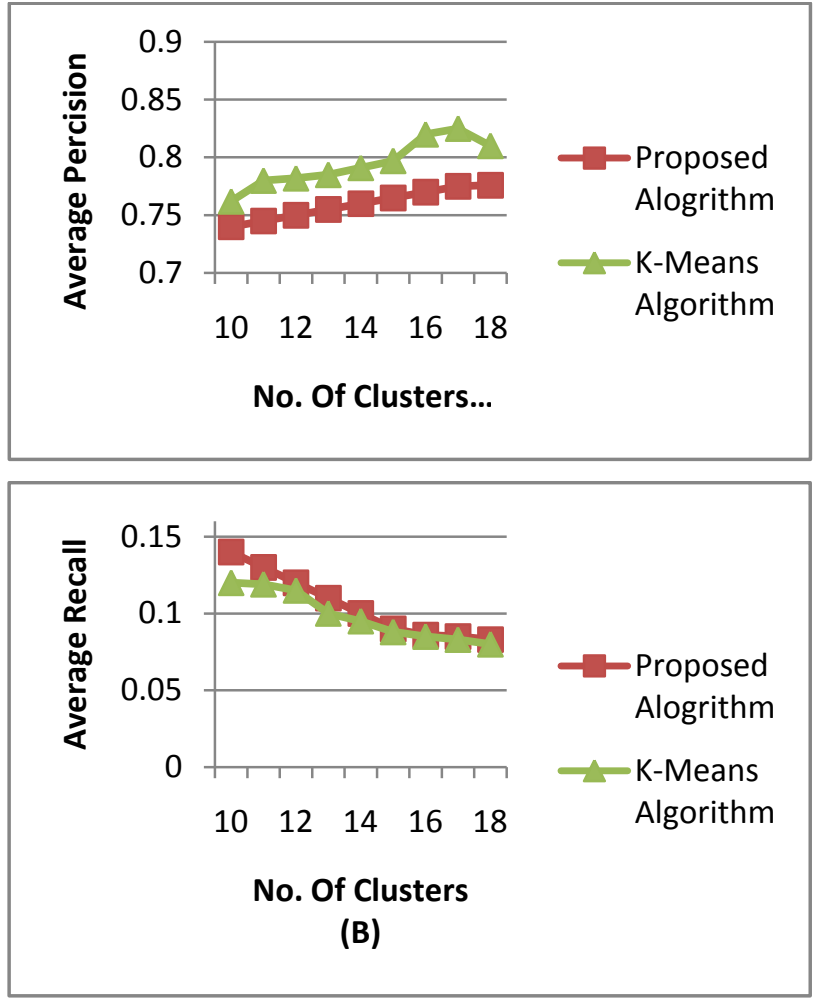

Fig.8. Average Precision and Recall against the number of clusters

The next table shows the classification accuracy using CBIR and k-Means segmentation techniques.

Table1. The Accuracy of the Classification Techniques

\begin{tabular}{|c|c|c|c|c|c|c|c|}
\hline $\begin{array}{c}\text { Segme } \\
\text { ntation }\end{array}$ & DA & NN & NB & SVM & DT & $\begin{array}{c}\text { KN } \\
\text { N }\end{array}$ & $\begin{array}{c}\text { TA } \\
\text { NN } \\
\text { N }\end{array}$ \\
\hline OASIS & 94.4 & 93.6 & 95.2 & 92.5 & 96.4 & $\begin{array}{c}96 . \\
6\end{array}$ & $\begin{array}{c}99 . \\
2\end{array}$ \\
\hline
\end{tabular}

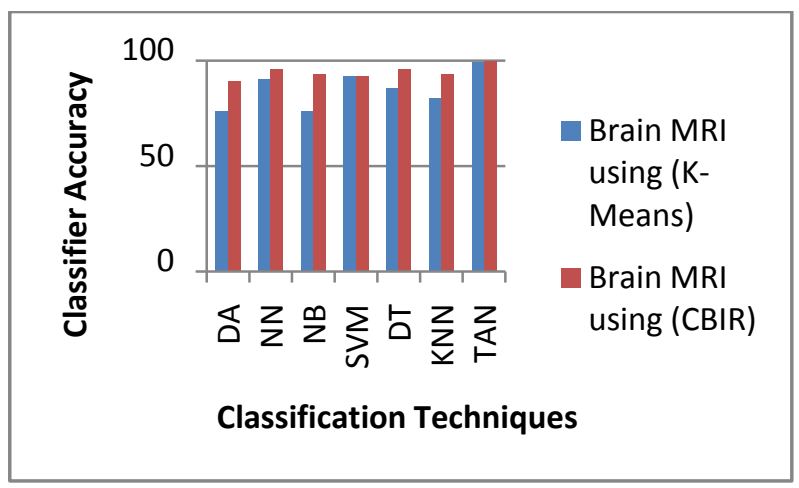

Fig.9. The accuracy of classification techniques.

The next table states a comparison between the classification techniques according to the classification time see table 2 .

Table2. The classification Techniques Classification Time

\begin{tabular}{|c|c|}
\hline $\begin{array}{c}\text { Classification } \\
\text { Technique }\end{array}$ & Classification Time (seconds) \\
\hline OASIS dataset & 1.9 \\
\hline
\end{tabular}

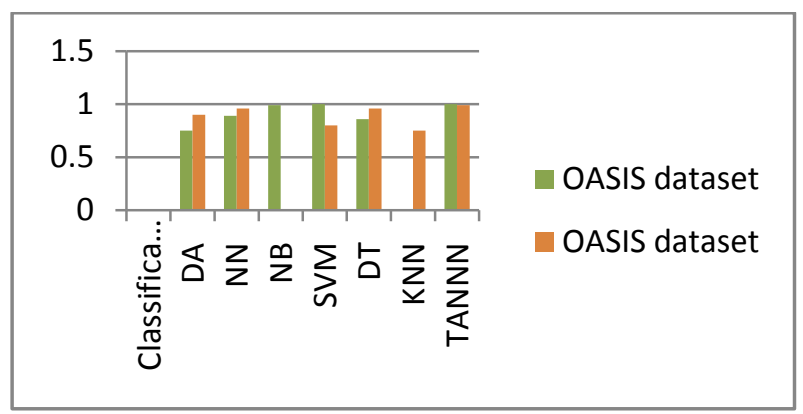

Fig.12. Classification results in terms of specificity and sensitivity

From the results of experiments, the decision tree algorithm gave the best detection rate. It, achieving a classification rate of $96.19 \%$. But when considering computational performance, however, K-Nearest Neighbor algorithm proved to have a faster build time (Time it takes to build a model on network training data) at 0.03 seconds while having a detection rate of $93.7 \%$ as shown in table 1. Naïve Bayes had the second best build time at 1.6 seconds but a detection rate of $93.52 \%$. Computational performance is particularly important when considering the real-time classification of potentially thousands of simultaneous networks traffic. From experiments, TANNN appears to be the best suited for realtime classification tasks due to its relatively fast classification speed and high detection rate.

\section{CONCLUSION}

This paper presents a survey on various image mining techniques that was proposed earlier by researchers for the better development in the field of content-based image retrieval. The purpose of the mining is to produce all considerable patterns without prior knowledge of the patterns. Important information can be hidden in images, conversely, few research talks about data mining on them. Image segmentation is the primary phase in image mining. In other words, image mining is simply an expansion of data mining in the field of image processing. Image mining handles with the hidden knowledge extraction, image data association and additional patterns which are not clearly accumulated in the images. Also, this paper provides a marginal overview for future research and improvements. Certain possible future investigations that are discussed may be done in the area of image mining which included the experimentations on other image elements such as textures, shape, etc.

In future, this program can be done more advanced so that Alzheimer can be classified according to its type. Also, Alzheimer growth can be analyzed by plotting the graph which can be obtained by studying sequential images of Alzheimer affected patient.

The future research work may include the implementation of the Bayesian networks for relevance feedbacks and more extensive tests with other examples of image forensic work. It is also envisaged that subjective testing will be performed with input from forensic experts.

Some possible future studies that may be conducted in the area of image mining include the experimentations on other image elements such as textures, shape, and so forth. It will also be interesting to investigate hidden relationships among images. For example, intensive and extensive exploratory 
pattern analysis involved in the existing systems in the database can be very useful.

\section{ACKNOWLEDGMENT}

I am very grateful and would like to thank my guides Prof. Mohammed Haggag and Prof. Ahmed Farag for their advice and continued support. Without them, it would not have been possible for me to complete this paper. I would like to thank my husband for the thoughtful and mind stimulating discussion we had, which prompted us to think beyond the obvious.

\section{REFERENCES}

[1] E. M. Ali, A. F. Seddik. M. H. Haggag, "Using Data Mining Techniques for children Alzheimerclassification based on MRI", International Journal of Computer applications , Pp. 36-42, Vol. 131 , No. 2 , December 2015.

[2] E. M. Ali, A. F. Seddik. M. H. Haggag, "Classification of Hydrocephalus using TAN", International Journal od Advanced Research in Computer Science and Software Engineering, Pp. 90-97, Vol. 5 , Issue. 11, November 2015.

[3] A. Rajkumar, "A Multi- Stage Hybrid , CAD Approach for MRI Brain Alzheimer Recognition and Classification", The IIOAB Journal School of Computing Science and Engineering VIT University, Januar 2016, India.

[4] S. Ganesh, "A Comparative Study on Various Brain Alzheimer Classification Methods", India Journal of Engineering, Vol. 13 , Pp. 27-33, January 2016.

[5] K. Sakthivel, B. Swathi, S. Vishnu, "Analysis of Medical Image Processing and it's Application in healthcare", International Journal od Advanced Engineering Research and Science (IJAERS), Vol. 3 Issue 2, Feb. 2016.

[6] Y. Li , Y. Mingquan, Z. Hao, "Alzheimer Diagnosis Based on the GMM Feature Decision Classification of
Brain MR Images", International journal of Multimedia and Ubiquitous Engineering, Vol. 11 No. 3 , Pp. 37- 44, 2016.

[7] K. Mayuri, R. Khode, S. Salwe, "A review on efficient Brain Alzheimer Detection Using Various Methods", International Journal of Research in Advent Technology , Vol. 4 , No. 2 , February 2016.

[8] L. Hou, D. Samaras, T. Kurc , " Patch-based Convolutional Neural Network for Whole Slide Tissue Image Classification", ARXIV , March 2016.

[9] N. Kasat, S. Thepade, "Novel Content Based Image Classification Method Using LBG Vector Quantization Method with Bayes and Lazy Family Data Mining Classifier", 7 th international conference on Communication, Computing and Virtualization, Pp. 483 $-489,2016$.

[10] G. Santhosh , K. Sivanaruleselvan, P. Betty, " Survey on Brain Tumour Detection and Classification Using Image Processing", ELK ASIA Pacific Journal of Computer Science and Information Systems, Vol. 2, Issue. 1, 2016.

[11] A. Mali, S. Pawar, "Detection \& Classification of Brain Alzheimer", International Journal of Innovative Research in Computer and Communication Engineering, Vol 4, Issue 1, January 2016.

[12] K. Selva , P. Geetha, "Semantic Feature Based Classification of Brain MRI using PCA and PNN", International Conference on Electrical, Electronics, and Optimization Techniques (ICEEOT), 2016.

[13] BRATS brain Alzheimer dataset, www.brainAlzheimeregmentation.org

[14] NBTR brain Alzheimer dataset, www.nbtr.nhs.uk

[15] OASIS brain Alzheimer dataset, www.oasis-brains.org/

[16] ADNI brain Alzheimer dataset, www.adni.loni.ucla.edu/ 